\title{
Nonadiabatic Molecular Dynamics by Multiconfiguration Pair-Density Functional Theory
}

\author{
Paul B. Calio, ${ }^{1}$ Donald G. Truhlar ${ }^{2 *}$, Laura Gagliardi ${ }^{3,4 *}$
}

${ }^{1}$ Department of Chemistry, Chicago Center for Theoretical Chemistry, James Franck Institute, University of Chicago, Chicago, Illinois

${ }^{2}$ Department of Chemistry, Chemical Theory Center, and Minnesota Supercomputing Institute, University of Minnesota, Minneapolis, Minnesota

${ }^{3}$ Department of Chemistry, Center for Theoretical Chemistry, Pritzker School of Molecular Engineering, Chicago James Franck Institute, University of Chicago, Chicago, Illinois

${ }^{4}$ Argonne National Laboratory, Lemont, Illinois

ABSTRACT. We present the first implementation of multiconfiguration pair-density functional theory (MC-PDFT) ab initio molecular dynamics. MC-PDFT is a multireference electronic structure method that in many cases has a similar accuracy (or even better accuracy) than complete active space second order perturbation theory (CASPT2) at a significantly lower computational cost. In this work we introduced MC-PDFT analytical gradients into the SHARC molecular dynamics program for $a b$ initio, nonadiabatic molecular dynamics simulations. We verify our implementation by examining the intersystem crossing dynamics of thioformaldehyde, and we observe excellent agreement with recent CASPT2 and experimental findings. Moreover, with MCPDFT we could perform dynamics with an active space that was computationally too expensive for CASPT2.

\section{Introduction}

Molecular dynamics calculations based on forces calculated directly from quantum mechanical electronic structure calculations ${ }^{1}$ have become a valuable tool for simulations of nuclear dynamics in chemical systems. In Born-Oppenheimer molecular dynamics (BOMD), ${ }^{2-10}$ based on the Born-Oppenheimer separation of electronic and nuclear motion, ${ }^{11}$ nuclear dynamics are restricted to a single electronic adiabatic state. Nonadiabatic molecular dynamics ${ }^{12-16}$ 
(NAMD) involves population transfer among electronic states, which is necessary to study internal conversion, intersystem crossing, and most photochemical processes. ${ }^{17}$

Many excited electronic states are inherently multiconfigurational, and near conical intersections, which are ubiquitous, ${ }^{18}$ excited states are always inherently multiconfigurational. Inherently multiconfigurational states are also called strongly correlated. Reliable electronic structure calculations on strongly correlated states require multiconfigurational reference wave functions ${ }^{19}$ (such calculations are called multireference calculations). The most common method to generate reference functions for multireference methods is complete active space selfconsistent field ${ }^{20}$ (CASSCF) theory, but this method does not capture correlation energy external to the active space and is therefore quantitatively unreliable when used without a post-SCF step like second order complete active space perturbation theory ${ }^{21-22}$ (CASPT2). The computational cost of CASPT2 and other multireference perturbation theory methods, although practical with analytic gradients ${ }^{23}$ for some cases,${ }^{24}$ makes quantitative calculations prohibitively expensive as the active space and system size increase.

An alternative post-SCF method is multiconfiguration pair-density functional theory ${ }^{25-26}$ (MC-PDFT), which has been shown to be in many cases as accurate as CASPT2 (or even more accurate in some cases) but at a lower computational cost. ${ }^{27}$ MC-PDFT computes the electron correlation by using a multireference wave function and a functional of the electron density and the on-top density, where the latter describes the probability of finding two electrons on top of each other at a given position in space. Analytical gradients (as required for efficient computation of forces on nuclei) have recently been developed for state-specific ${ }^{28}$ and stateaveraged $^{29-30}$ MC-PDFT. This allows us to expand the application of MC-PDFT from calculating static properties via single-point calculations ${ }^{31}$ to studying dynamical properties of strongly correlated systems. This is in principle more accurate than using time-dependent density functional theory ${ }^{32-33}$ (TD-DFT) or CASSCF CA-36 $^{34 c a u s e ~ T D-D F T ~ u s e s ~ a ~ s i n g l e-c o n f i g u r a t i o n ~}$ reference and CASSCF lacks external correlation.

In this work, we present the first application of MC-PDFT for molecular dynamics. In particular we present an application to nonadiabatic molecular dynamics by implementing MCPDFT nuclear gradients into the SHARC $C^{37-39}$ molecular dynamics program. One strong feature of SHARC is that it treats internal conversion and intersystem crossing on the same footing using a combination of spin-orbit-free input energies, gradient, and nonadiabatic couplings plus spin- 
orbit matrix elements in a spin-orbit-free electronic basis, and we are able to supply this information using our implementation of MC-PDFT in OpenMolcas. ${ }^{40-41}$ The application presented here is the intersystem crossing dynamics of thioformaldehyde $\left(\mathrm{CH}_{2} \mathrm{~S}\right)$ after it is excited into the $S_{1}$ electronic state. Thioformaldehyde is a simple molecule that has been used to understand fundamental trends of carbonyls, and rates of radiationless transitions. ${ }^{42}$ El-Sayed's propensity rule ${ }^{43}$ states that intersystem crossing usually occurs more rapidly if the transition is between orbitals of different symmetry. This implies that the intersystem crossing rate of thioformaldehyde from the $\mathrm{S}_{1}$ electronic state, which is a $n \rightarrow \pi^{*}$ transition, will populate the $\mathrm{T}_{2}$ $\left(\pi \rightarrow \pi^{*}\right)$ state in preference to the $\mathrm{T}_{1}\left(n \rightarrow \pi^{*}\right)$ state. However less probable events like the $\mathrm{S}_{1}$ to $\mathrm{T}_{1}$ transitions can and have been observed in molecular simulations. ${ }^{44}$

Thioformaldehyde has previously been studied with the SHARC program, but with other electronic structure methods, ${ }^{24,44}$ and its small size allows us to compare our results to those obtained with more expensive methods. The intersystem crossing rate for thioformaldehyde has not been measured experimentally to compare with MC-PDFT. However, Mai et. al. ${ }^{24}$ have proposed that the intersystem crossing rate for thioformaldehyde is small due to the large fluorescence yields that have been measured experimentally, ${ }^{45-47}$ and this conclusion is corroborated by their simulations. ${ }^{24,44}$

Mai et al. ${ }^{24}$ and Zhang et. al. ${ }^{44}$ investigated the intersystem crossing dynamics of thioformaldehyde using the $S H A R C$ surface hopping procedure. Mai et al. ${ }^{24}$ compared various electronic structure methods for the problem studied here. Of the methods used for dynamics, it was concluded that MS-CASPT2 $(10,6)$ [where $(\mathrm{x}, y)$ denotes $x$ active electrons in $y$ active orbitals] gave the most accurate results for the following reasons: (i) It predicted good vertical excitations in agreement with MS-CASPT2(12,10) and multireference configuration interaction with single and double excitations with the Pople size-extensity correction [MRCISD+P $(12,10)^{48-49}$ ] (these methods were, however, too expensive to be used for dynamics). (ii) It predicted potential energy curves outside the Frank-Condon region in good agreement with MS-CASPT2 $(12,10)$ and MR-CISD+P(12,10). (iii). It showed no intersystem crossing dynamics on a femtosecond timescale. They also used $\operatorname{CASSCF}(10,6)$ as the electronic structure method for dynamics, but these simulations gave a 5\% excited state population transfer within $500 \mathrm{fs}$ due to $\operatorname{CASSCF}(10,6)$ underestimating the $\mathrm{T}_{2}-\mathrm{S}_{1}$ energy gaps. Zhang et. al. ${ }^{44}$ investigated various decoherence schemes with $\operatorname{CASCCF}(12,10)$ and observed a reduced population transfer in 
thioformaldehyde in comparison to the $\operatorname{CASSCF}(10,6)$ simulation of Mai et. al. ${ }^{24}$ Neither study used MS-CASPT2(12,10) for dynamics due to its computational expense. Here, we show thioformaldehyde intersystem crossing dynamics results for MC-PDFT with both the $(10,6)$ and $(12,10)$ active spaces; we find energetic and dynamical results that agree with Mai et al.'s MSCASPT2 $(10,6)$ results and that further support experimental findings.

The paper is organized as follows. In Section 2, we introduce the electronic structure calculations to be used for dynamics and for calculations done prior to doing dynamics. In Section 3, we discuss haw SHARC carries out dynamics and how electronic structure data is provided to $S H A R C$. In Section 4, we outline the simulation methods. We then present the results and discussion in Section 5, and we provide our conclusions and outlook in Section 6.

\section{Electronic structure calculations}

Calculations were performed for the two lowest singlet states $\left(\mathrm{S}_{0}\right.$ and $\left.\mathrm{S}_{1}\right)$ and the two lowest triplet states $\left(\mathrm{T}_{1}\right.$ and $\left.\mathrm{T}_{2}\right)$ of thioformaldehyde. In the first step, electronic structure calculations were carried out by state-averaged (SA)-CASSCF and MC-PDFT with the tPBE ontop functional (MC-PDFT/tPBE) using OpenMolcas v21.02. One set of calculations was state averaged over the two lowest singlet states $\left(\mathrm{S}_{0}\right.$ and $\left.\mathrm{S}_{1}\right)$, and another set of calculations was state averaged over the two lowest triplet states $\left(\mathrm{T}_{1}\right.$ and $\left.\mathrm{T}_{2}\right)$. The resulting CASSCF functions serve as the reference wave functions for MC-PDFT calculations in which each of the four states is treated separately.

All SA-CASSCF and MC-PDFT calculations were performed with a cc-pVDZ basis set, ${ }^{50}$ the Douglas-Kroll-Hess Hamiltonian, ${ }^{51-53}$ and either a $(10,6)$ or $(12,10)$ active space. Figure 1 shows the state-averaged orbitals of the singlet state used in the $(12,10)$ active space of thioformaldehyde's optimized structure. We show the orbitals for the $(10,6)$ active space in Figure S1 (figures and tables with the prefix "S" are in Supporting Information.). The MC-PDFT gradients based on a SA-CASSCF wave function are described elsewhere. ${ }^{14}$ 


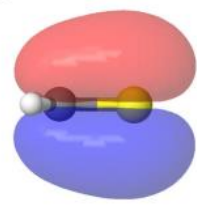

$\pi$

1.906

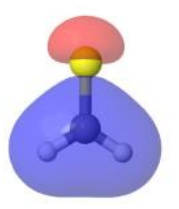

$\sigma_{C-H}$

1.979

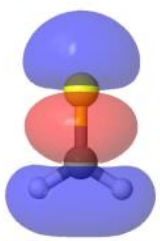

$\sigma_{C-S}$

1.972

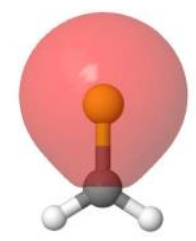

$3 \mathrm{~s}$

1.997

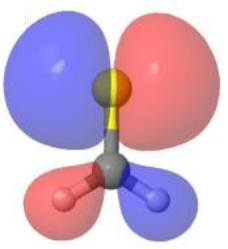

n

1.499

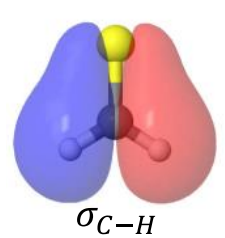

1.974

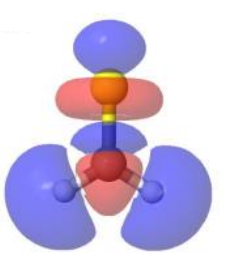

$\sigma_{C-H}^{*}$

0.023

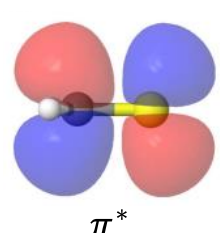

0.595

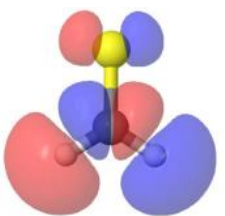

$\sigma_{C-H}^{*}$ 0.024

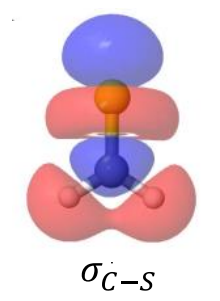

0.030

Figure 1. State-averaged natural orbitals and their state-averaged occupation numbers for the two singlet states as calculated by SA-CASSCF with the $(12,10)$ active space. All MOs were plotted using a cutoff of 0.025 a.u.

Before running dynamics, we obtained the optimized structures in the singlet ground state for SA-CASSCF and MC-PDFT/tPBE with both active spaces (the resulting structures and absolute energies are in Tables S1-S3). After optimizing the structures, vertical excitations from the $S_{0}$ electronic state into the $S_{1}, T_{1}$, and $T_{2}$ were computed, and the results will be given in Section 5.1.

The dynamics calculations also require the spin-orbit matrix elements. Because the singlet and triplet SA-CASSCF calculations produce different orbitals, these matrix elements were calculated using a biorthogonal procedure ${ }^{54}$ in the RASSI module of OpenMolcas together with an effective one-electron spin-orbit Hamiltonian based on atomic mean field integrals. ${ }^{55}$

The dynamics calculations also require the nonadiabatic coupling vectors and these were evaluated in the SA-CASSCF approximation using OpenMolcas procedures described elsewhere. ${ }^{56}$

\section{Theory}

\subsection{Dynamics Method}

Dynamics calculations were carried out with a locally modified version of SHARC v2.1. The population dynamics of the triplet states in thioformaldehyde has previously been studied with fewest-switches trajectory surface hopping (FS-TSH), ${ }^{57}$ with the semiclassical Ehrenfest 
method, ${ }^{58-59}$ with fewest-switches trajectory surface hopping with energy-based decoherence ${ }^{60-61}$ (FS-TSH-EDC), and with coherent switching with decay of mixing ${ }^{62}$ (CSDM). These methods are all semiclassical in that the electronic structure is treated quantum mechanically by the timedependent Schrödinger equation, and the nuclei are propagated by trajectories governed by multiple potential energy surfaces; however, the former two methods do not include decoherence and the latter two methods do. In previous studies of thioformaldehyde triplet dynamics, ${ }^{44}$ it was found that deoherence plays an important role, and the two latter methods agree well with one another. Therefore, in the present work we selected one of the latter two methods, FS-TSH-EDC. The decoherence in the FS-TSH-EDC method ${ }^{60-61}$ is based on an earlier approximation ${ }^{62}$ to the decoherence time in terms of energy gaps and nuclear kinetic energies

The total Hamiltonian for intersystem crossing dynamics in SHARC is written as

$$
H^{\text {total }}=H^{M C H}+H^{S O C}
$$

where $H^{\mathrm{MCH}}$ is the molecular Coulombic Hamiltonian (electronic kinetic energy plus Coulomb interactions), and $H^{\mathrm{SOC}}$ is the spin-orbit coupling operator. SHARC treats the dynamics in terms of two basis sets. The first basis is the set of eigenstates of $H^{\mathrm{MCH}}$; we call this spin-orbit-free basis (also called the spin-orbit-free representation); the total electron spin $S$ and its component $M_{S}$ are good quantum numbers in this basis. We use $\psi_{\mu}$ and $\psi_{\nu}$ to represent spin-orbit-free electronic states such as a singlet state $\left(S_{0}\right)$ or a triplet state with $M_{S}$ specified by a superscript $\left(T_{1}^{-1}, T_{1}^{0}\right.$, or $\left.T_{1}^{1}\right) . H^{\mathrm{MCH}}$ is diagonal in this basis with diagonal elements $E_{\mu}$, and the spin-orbit coupling has diagonal elements equal to zero and off-diagonal elements that can be nonzero. Therefore,

$$
\begin{gathered}
H_{\mu \nu}=E_{\mu} \delta_{\mu \nu}+C_{\mu \nu} \\
E_{\mu}=\left\langle\psi_{\mu}\left|H^{\mathrm{MCH}}\right| \psi_{\mu}\right\rangle \\
C_{\mu \nu}=\left\langle\psi_{\mu}\left|H^{\mathrm{SOC}}\right| \psi_{\nu}\right\rangle
\end{gathered}
$$

The nonadiabatic coupling vector in the spin-orbit-free representation is given by

$$
\mathbf{d}_{\mu \nu}=\left\langle\psi_{\mu}|\nabla| \psi_{\nu}\right\rangle \text {. }
$$

where $\nabla$ is a $3 N$-dimensional gradient, and $N$ is the number of atoms.

The dynamics calculations are carried out in the diagonal basis (which may also be called the fully adiabatic basis, the spin-mixed basis, or the spin-orbit-coupled basis). This is the basis 
in which the total Hamiltonian is diagonal. We use $\psi_{\alpha}$ and $\psi_{\beta}$ to represent the basis functions in the diagonal basis, which are the eigenvectors of $H^{\text {total }}$. An FS-TSH-EDC dynamics calculation in this basis requires the eigenvalues $E_{\alpha}$, their gradients $\nabla E_{\alpha}$, and the time matrix elements $\sigma_{\alpha \beta}$ in the diagonal representation, given along a trajectory by

$$
\sigma_{\alpha \beta}=\left\langle\psi_{\alpha}\left|\frac{d}{d t}\right| \psi_{\beta}\right\rangle
$$

Note that

$$
\sigma_{\alpha \beta}=\left\langle\psi_{\alpha}|\nabla| \psi_{\beta}\right\rangle \cdot \mathbf{v}
$$

where $\mathbf{v}$ is the instantaneous $3 N$-dimensional nuclear velocity vector of the trajectory.

$S H A R C$ requires only four kinds of data: the spin-orbit-free eigenvalues $E_{\mu}$, their gradients $\nabla E_{\mu}$, the spin-orbit coupling matrix elements $C_{\mu \nu}$ in the spin-orbit-free basis, and the nonadiabatic coupling vectors $\mathbf{d}_{\mu \nu}$ in the spin-orbit-free representation. SHARC translates these to the quantities needed in the diagonal basis with only one approximation, namely it neglects $\left\langle\psi_{\mu}\left|\nabla H^{\mathrm{SOC}}\right| \psi_{v}\right\rangle$ in the transformation of the gradients. The equations are given elsewhere. ${ }^{37-39}$

The approximation in the transformation of the gradients could in principle cause poor conservation of energy in SHARC trajectories. However, a previous study of thioformaldehyde using FS-TSH-EDC with SHARC showed that the overall total energy conservation within the SHARC gradient approximation is good enough for the results to be meaningful for this system.

\subsection{Electronic structure input}

As explained in Section 2.1, we need to input $E_{\mu}, \nabla E_{\mu}, C_{\mu \nu}$ and $\mathbf{d}_{\mu \nu}$ in the spin-orbit-free representation $\left(\psi_{\mu}\right.$ and $\left.\psi_{v}\right)$. The diagonal representation in this study is the MC-PDFT one, and this uses the $E_{\mu}$ and $\nabla E_{\mu}$ from MC-PDFT, but the $C_{\mu \nu}$ and $\mathbf{d}_{\mu \nu}$ are approximated at the SASCASSCF level.

The approximation of the nonadiabatic coupling vector at the CASSCF level is an approximation that can be good only when the dynamics is not dominated by passage near conical intersections, and that condition is satisfied in the present application. In the more general case one would need to calculate the nonadiabatic coupling vector in a multi-state approximation for two reasons: (i) because the inclusion of external correlation will change the locations where the conical intersections occur and hence where the large peaks in the nonadiabatic coupling vector occur, and (ii) because one must use a multi-state approximation 
near conical intersections. In such a case, the multistate nonadiabatic coupling vector can be calculated by a Lagrangian method. ${ }^{63-64}$

\section{Details of the simulation}

We calculate the dynamic evolution of thioformaldehyde after exciting the molecule into the $S_{1}$ electronic state. We prepared 10,000 initial conditions from the Wigner distribution ${ }^{65}$ using SA-CASSCF $(10,6)$ or SA-CASSCF $(12,10)$ harmonic frequencies to sample the ground state potential energy well (the harmonic frequencies are in Table S4). Excitation energies for SA-CASSCF and MC-PDFT were then used in conjunction with the CASSCF transition-dipole moments to randomly select initial conditions for the simulations using the procedure of Barbatti et. al. ${ }^{66}$ Of those prepared, 250 initial conditions were propagated using SHARC dynamics for each of four methods: CASSCF(10,6), MC-PDFT(10,6), CASSCF(12,10), and MC$\operatorname{PDFT}(12,10)$.

The simulation involves eight electronic states in the diagonal basis, two from the singlets and six from the two triplets. All simulations ran for a minimum of $500 \mathrm{fs}$ using a nuclear timestep of $0.5 \mathrm{fs}$ and an electronic timestep of $0.02 \mathrm{fs}$. The local diabatization method ${ }^{67}$ was used for coupling states of the same multiplicity. Nonadiabatic couplings were used to transform the gradients from the $\mathrm{MCH}$ representation to the diagonal representation. Out of the 250 trajectories, 203, 193, 249, and 198 trajectories successfully completed for $\operatorname{CASSCF}(10,6), \mathrm{MC}$ PDFT(10,6), CASSCF(12,10), and MC-PDFT(12,10), respectively. Of the simulations that successfully completed, three of the $\operatorname{SA}-\operatorname{CASSCF}(10,6)$ simulations transitioned from the $\mathrm{S}_{1}$ to the $\mathrm{T}_{2}$ state. Because these transitions are rare events for which meaningful statistics were not obtained, we removed them from the analysis.

\section{Results and discussion}

\subsection{Vertical excitation energies of thioformaldehyde}

Table 1 presents the $S_{1}, T_{1}$, and $T_{2}$ vertical excitation energies for the SA-CASSCF and MC-PDFT methods. Our benchmark values will be the averages of the MRCISD+P $(12,10)^{48-49}$ and MS-CASPT2 $(12,10)^{22,68}$ calculations of Ref. ${ }^{24}$ because only $0-0$ transitions are available experimentally. ${ }^{45}$ Table 1 reports these values and also shows the MS-CASPT2(10,6) vertical excitations of Ref. ${ }^{24}$ to compare with our MC-PDFT/tPBE results. The table also gives the rootmean-squared deviation of the various excitation energies from the $\mathrm{S}_{0}$ state, denoted as 
$\operatorname{RMSD}(\Delta \mathrm{E})$, and the root-mean-squared deviation of the energy differences among all the excited states, denoted as $\operatorname{RMSD}(\Delta \Delta \mathrm{E})$.

Table 1 shows that of the present calculations, $\operatorname{SA}-\operatorname{CASSCF}(10,6)$ has the largest deviations for the benchmark values, $0.22 \mathrm{eV}$ and $0.36 \mathrm{eV}$, respectively. The SA-CASSCF $(10,6)$ vertical excitation energies are in close agreement with previously reported values for this level of calcualtion. ${ }^{24}$ However, the table shows that MC-PDFT using the same $(10,6)$ active space reduces the $\operatorname{RMSD}(\Delta \mathrm{E})$ by half and the $\operatorname{RMSD}(\Delta \Delta \mathrm{E})$ by a factor of seven, and it closely matches the MS-CASPT2 $(10,6)$ calculations of Mai et al. ${ }^{24}$

The table also shows that increasing the active space to $(12,10)$ improves the accuracy of the MC-PDFT vertical excitation energies by $25 \%$ but slightly worsens the accuracy of the energy differences.

Of special importance among the energy differences is the $\mathrm{T}_{2}-\mathrm{S}_{1}$ gap because it has the greatest effect on the intersystem crossing dynamics. ${ }^{24}$ Although the population dynamics is governed by the potentials along the whole trajectory and not just at the Frank-Condon point, ${ }^{24}$ the difference in vertical excitation energies is our best indication of the accuracy of the gap, and we see that SA-CASSCF $(10,6)$ underestimates the vertical gap by $0.45 \mathrm{eV}$, which makes the dynamics with this method unreliable. The two MC-PDFT values of the vertical $\mathrm{T}_{2}-\mathrm{S}_{1}$ gap have deviations from the benchmark of only 0.01 and $0.09 \mathrm{eV}$. Figure S2 presents the potential energy curve along the C-S bond for the four methods used in this study.

Table 1. Excitation energies, the vertical $\mathrm{T}_{2}-\mathrm{S}_{1}$ gap, and deviations from benchmark (in eV)

\begin{tabular}{|c|c|c|c|c|c|c|}
\hline Method & $\mathbf{S}_{1}$ & $\mathbf{T}_{1}$ & $\mathbf{T}_{2}$ & $\mathbf{T}_{2}-\mathbf{S}_{1}$ & RMSD- $\Delta \mathbf{E}^{a}$ & RMSD- $\Delta \mathbf{\Delta} \mathbf{E}^{a}$ \\
\hline \multicolumn{7}{|c|}{ Previous work ${ }^{24}$} \\
\hline MRCISD+P(12,10) & 2.19 & 1.91 & 3.42 & 1.23 & & \\
\hline MS-CASPT2 $(12,10)$ & 2.25 & 2.00 & 3.45 & 1.20 & & \\
\hline Benchmark $^{b}$ & 2.22 & 1.96 & 3.44 & 1.22 & 0.00 & 0.00 \\
\hline MS-CASPT2 $(10,6)$ & 2.14 & 1.84 & 3.31 & 1.17 & 0.11 & 0.04 \\
\hline \multicolumn{7}{|c|}{ Present work } \\
\hline MC-PDFT(12,10) & 2.34 & 2.04 & 3.47 & 1.13 & 0.09 & 0.06 \\
\hline SA-CASSCF $(12,10)$ & 2.40 & 2.16 & 3.31 & 0.91 & 0.17 & 0.26 \\
\hline MC-PDFT $(10,6)$ & 2.35 & 2.03 & 3.58 & 1.23 & 0.12 & 0.05 \\
\hline $\operatorname{SA-CASSCF}(10,6)$ & 2.31 & 2.02 & 3.08 & 0.77 & 0.22 & 0.36 \\
\hline
\end{tabular}




\subsection{SHARC dynamics with SA-CASSCF and MC-PDFT}

Previous SHARC simulations of thioformaldehyde with multireference methods examined the intersystem crossing dynamics from the $S_{1}$ state into the $T_{1}$ and $T_{2}$ state. Mai et al. ${ }^{24}$ observed that simulations with MS-CASPT2 $(10,6)$ showed no intersystem crossing dynamics on a $500 \mathrm{fs}$ timescales, while $\mathrm{SA}-\mathrm{CASSCF}(10,6)$ showed a $5 \%$ population transfer to the $\mathrm{T}_{2}$ state in this timeframe. The carbon-sulfur bond stretch frequency was shown to impact the spin-orbit coupling between the $S_{1}$ and $T_{2}$ electronic states, and further manifested oscillations in the $\mathrm{MCH}$ population. Zhang et al. showed that dynamical results calculated with $\operatorname{SA}-\operatorname{CASSCF}(10,12)$ agree better with previous results using MS-CASPT2 than do dynamical results with SA$\operatorname{CASSCF}(10,6)$. (Note that the calculations of Zhang et al. ${ }^{44}$ were performed with a different SACASSCF scheme; in particular, they used a single set of orbitals and did not employ the biorthogonalization scheme used here.)

a)

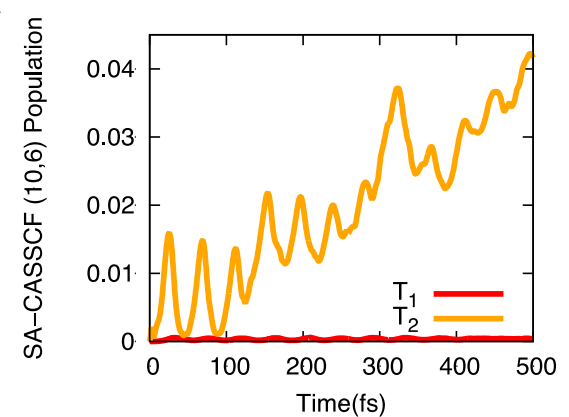

c)

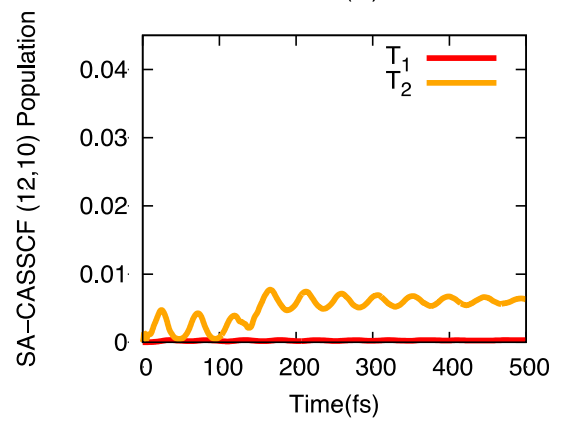

b)

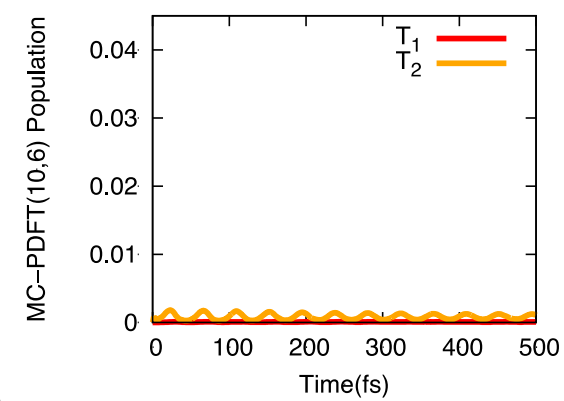

d)

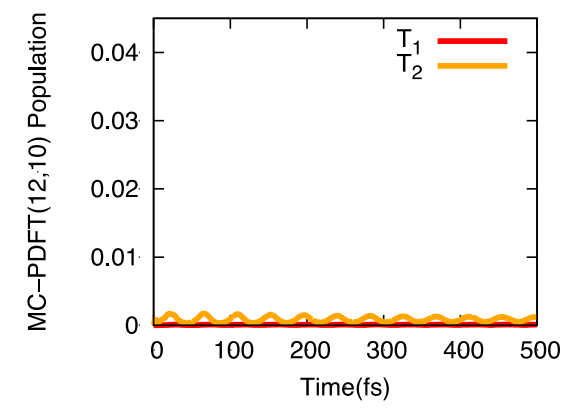

Figure 2. $\mathrm{T}_{1}$ (red) and $\mathrm{T}_{2}$ (orange) population according to the $\mathrm{MCH}$ quantum amplitudes for (a) SA-CASSCF(10,6), (b) MC-PDFT(10,6), (c) SA-CASSCF(12,10), and (d) MC-PDFT(12,10) 
Figure 2 shows the $\mathrm{T}_{1}$ and $\mathrm{T}_{2}$ populations for all four methods studied here. The SACASSCF $(10,6)$ results in panel a show a steady increase in the $\mathrm{T}_{2}$ population during the simulation, with a population transfer of about $4 \%$ within 500 fs. As been shown previously, ${ }^{24}$ this is due to $\operatorname{SA}-C A S S C F(10,6)$ underestimating the $\mathrm{T}_{2}-\mathrm{S}_{1}$ gap. MC-PDFT(10,6), Fig $2 \mathrm{~b}$, on the other hand shows no population transfer at the $(10,6)$ active space in agreement with previous MS-CASPT2 $(10,6)$ simulations; this is consistent with the good agreement in the $\mathrm{T}_{2}-\mathrm{S}_{1}$ energy gaps predicted by the two methods (Table 1 and Fig. S2).

Panels c and d of Fig. 2 show SA-CASSCF(12,10) and MC-PDFT(12,10) simulations, respectively. SA-CASSCF $(12,10)$ simulations have a reduced population transfer as compared to those with the $(10,6)$ active space in agreement with previous work. ${ }^{44}$ By simulating thioformaldehyde with MC-PDFT(12,10), we further reduce the population transfer into the $\mathrm{T}_{2}$ state.

The oscillations observed in the population dynamics have been ascribed to the C-S bond and its influence on the spin-orbit coupling between the $S_{1}$ and $T_{2}$ states. ${ }^{24,44,69}$ Figure 3 shows the $\mathrm{C}-\mathrm{S}$ bond distance in thioformaldehyde for each trajectory of the various electronic structure methods and the magnitude spin-orbit coupling value between the $\mathrm{S}_{1}$ state and the $\mathrm{T}_{2}$ manifold as calculated by

$$
|S O C|=\sqrt{\sum_{M_{S}=-1,0,1}\left|\left\langle S_{1}\left|H^{S O C}\right| T_{2}^{M_{S}}\right\rangle\right|^{2}}
$$

For all the methods, we observe strong correlation among the C-S bond lengths of the various trajectories, but this correlation becomes weaker as the simulations progress. For the SACASSCF simulations, the bond distance oscillates around a mean value of $1.8 \AA$, with a maximum bond distance of around $2.2 \AA$. For the MC-PDFT simulations, the average C-S bond distance is $\sim 1.7 \AA$, the maximum is $\sim 2.05 \AA$, and in some cases the bond length is as large as 2.1 $\AA$. We additionally see that $|S O C|$ follows the trends of the average C-S bond distance, and has an average value of $165 \mathrm{~cm}^{-1}$ and $163 \mathrm{~cm}^{-1}$ for the $(10,6)$ and $(12,10)$ active spaces, respectively. Interestingly, the SOC for SA-CASSCF and MC-PDFT are very similar, which is not surprising since the MC-PDFT calculations obtain the SOC matrix elements from the SA-CASSCF wave function. Nevertheless the CASSCF simulations have more population transfer from the $\mathrm{S}_{1}$ to the $\mathrm{T}_{2}$ state than the MC-PDFT simulations. We attribute this to the MC-PDFT energy and gradients. As the $\mathrm{C}-\mathrm{S}$ bond lengthens, the $\mathrm{T}_{2}-\mathrm{S}_{1}$ energy gap approaches zero in both MC-PDFT and SA- 
CASSCF (see Fig. S2); however, the MC-PDFT gradients cause the thioformaldehyde to sample smaller C-S bond distances where the $\mathrm{T}_{2}-\mathrm{S}_{1}$ energy gap is larger, while the CASSCF simulations sample larger $\mathrm{C}-\mathrm{S}$ bond distances where the $\mathrm{T}_{2}-\mathrm{S}_{1}$ energy gap is smaller, and this enhances the intersystem crossing. This is a very clear illustration of the importance of including external correlation in photochemical simulations.

a)

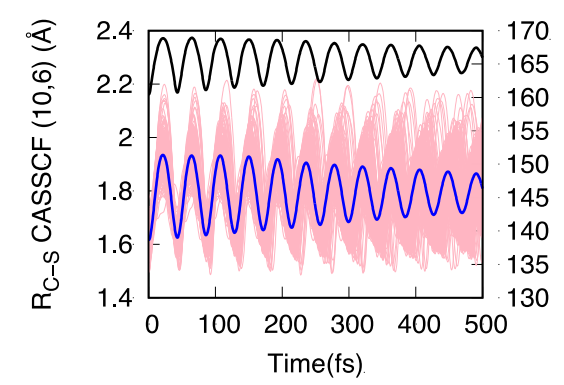

c)

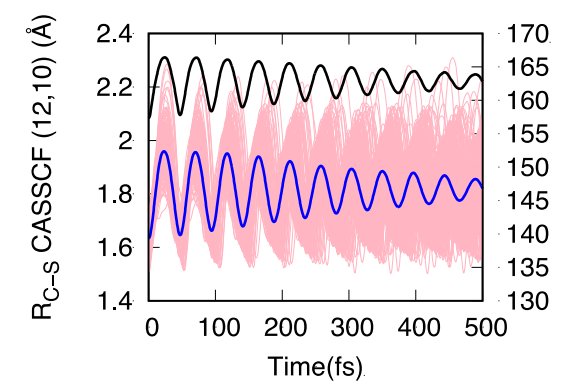

b)

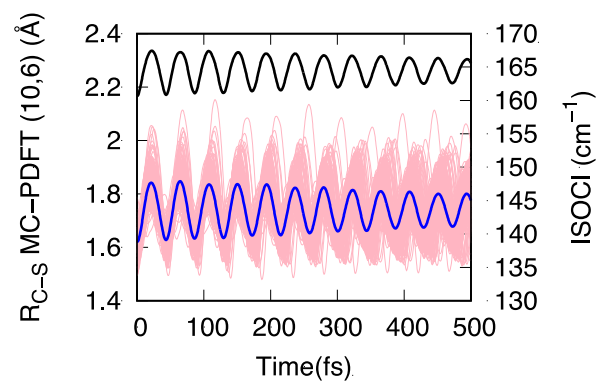

d)

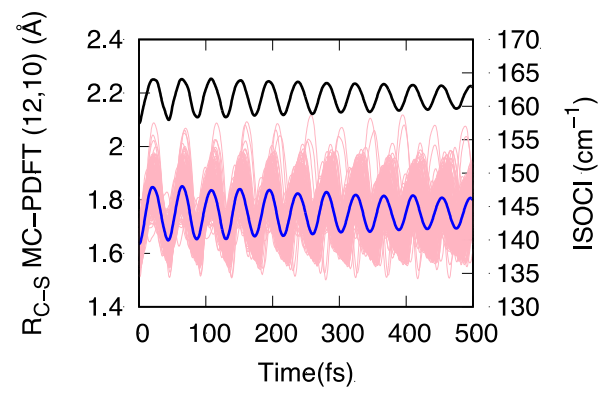

Figure 3. (left) C-S bond distance. (right) Magnitude of the $S_{1}$ coupling to the $T_{2}$ manifold. (a) SA-CASSCF(10,6), (b) MC-PDFT(10,6), (c) SA-CASSCF(12,10), (d) MC-PDFT(12,10). The C$\mathrm{S}$ bond distance for each trajectory is shown in pink, the average C-S bond distance is shown in blue, and the spin-orbit coupling is shown in black.

In this work, we have focused on running SHARC simulations with MC-PDFT for systems with intersystem crossing dynamics since the MC-PDFT gradients are based on MC-PDFT without state interaction. (State interaction can be included in various ways in MC-PDFT, ${ }^{70-72}$ and gradients using one of these methods, namely compressed-state multi-state PDFT, ${ }^{72}$ are under development.). To further verify that thioformaldehyde is an appropriate system for the current implementation of MC-PDFT, we show in Figure 4 the average potential energy for the two singlet and two triplet states. For both SA-CASSCF and MC-PDFT with the $(10,6)$ and $(12,10)$ active spaces, there is a clear separation between the $S_{0}$ and $S_{1}$ states and between the $T_{1}$ 
and $\mathrm{T}_{2}$ states, which confirms that the lack of state interaction does not make the current method inappropriate for the present application.

a)

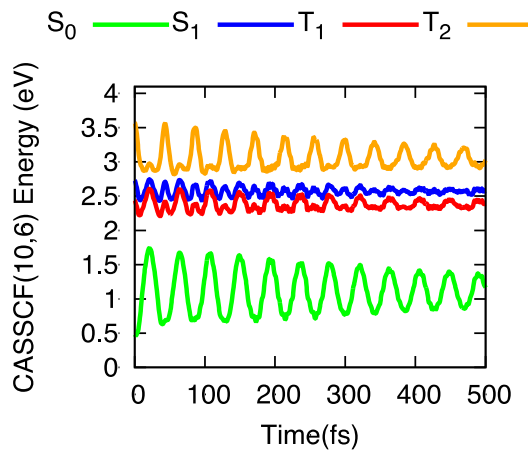

c)

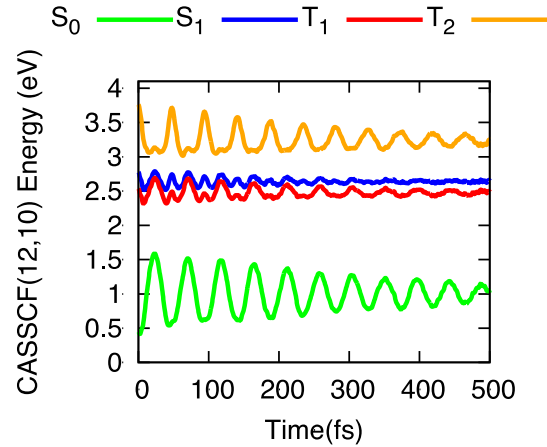

b)

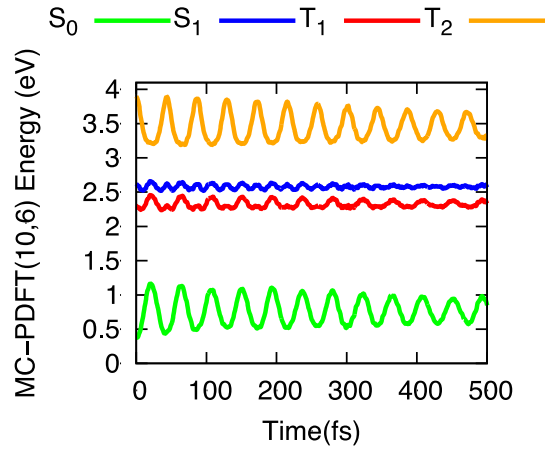

d)

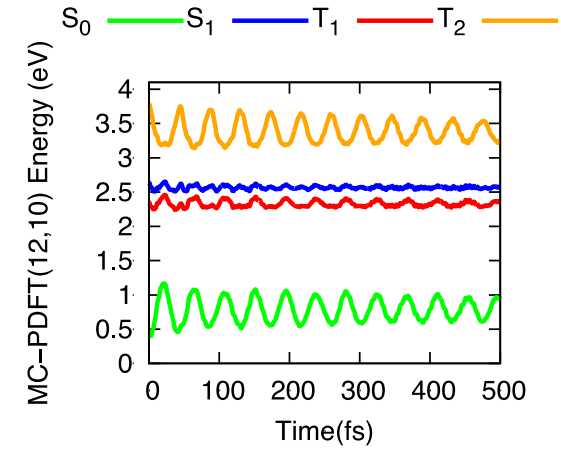

Figure 4. Average $S_{0}$ (green), $S_{1}$ (blue), $T_{1}$ (red), and $T_{2}$ (orange) potential energy for (a) SACASSCF(10,6), (b) MC-PDFT(10,6), (c) SA-CASSCF(12,10), and (d) MC-PDFT(12,10).

An advantage of MC-PDFT is its ability to obtain results comparable to MS-CASPT2 while being computationally more affordable than MS-CASPT2, an advantage that is especially relevant for large systems and large active spaces. Thioformaldehyde has not been simulated using MS-CASPT2 $(12,10)$ due to the computational cost. The timings for simulations with MCPDFT(12,10)/tPBE are presented in Table S5.

Further inspection of the SA-CASSCF $(12,10)$ simulations revealed a difference from the results in ref. ${ }^{44}$. This is shown in Fig. 5a, which is like Fig. 2c except that we zoomed from an ordinate scale of 0.04 in Fig. 2c to an ordinate scale of 0.08 in Fig. $5 b$. Th difference is due to a single trajectory, shown in Fig. 5b, that hopped from the $S_{1}$ to the $T_{2}$ state around $150 \mathrm{fs}$, which explains the mean population shift from $\sim 0.2$ to $\sim 0.6$ at 150 fs in Fig 5a. Zhang et. al.'s SACASSCF(12,10)/6-31G* SHARC simulations with energy-based decoherence correction showed a decaying oscillation during the 500 fs simulation without any hopping. ${ }^{44}$ These simulations used nuclear and electronic timesteps of 0.1 and $0.0005 \mathrm{fs}$, respectively; these are smaller 
timesteps than the one used in the present work. In Fig 5b, we investigate the effects of nuclear and electronic timestep on the $\mathrm{T}_{2}$ population of the trajectory that hopped. At early times $(<150$ fs), the two $\mathrm{T}_{2}$ populations align perfectly, but around $150 \mathrm{fs}$, the trajectory using the larger timestep ( 0.5 fs nuclear timestep and 0.02 fs electronic timestep) transitioned into the $\mathrm{T}_{2}$ state, while the simulation using the smaller timestep $(0.1 \mathrm{fs}$ nuclear timestep and 0.0005 fs electronic timestep) continued in the $\mathrm{S}_{1}$ state. We note that if a system requires a smaller timestep to accurately model the dynamics, then the cost advantage of MC-PDFT over MS-CASPT2 becomes even more important when choosing the electronic structure method.

a)

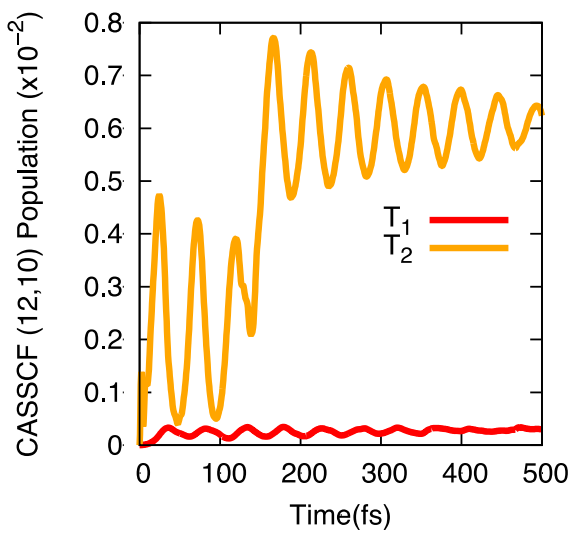

b)

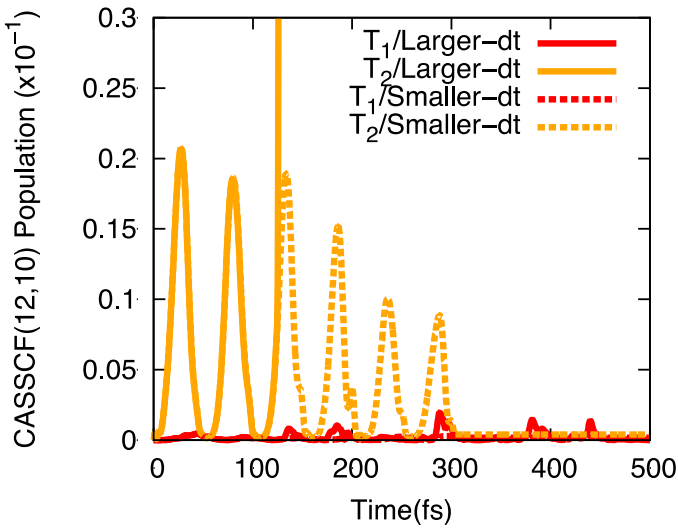

Figure 5. (a) Zoomed in plot of the SA-CASSCF(12,10) $\mathrm{T}_{1}$ (red) and $\mathrm{T}_{2}$ (orange) mean populations from the quantum amplitudes in the spin-uncoupled basis using the nuclear and electronic timesteps of 0.5 and 0.02 fs respectively. (b) $\operatorname{SA}-\operatorname{CASSCF}(12,10) \mathrm{T}_{1}$ and $\mathrm{T}_{2}$ populations of a single trajectory that had a $S_{1}$ to $T_{2}$ transition at $150 \mathrm{fs}$ when using the timesteps of $0.5 \mathrm{fs}$ and $0.02 \mathrm{fs}$ as used in panel a and when using the smaller time steps of 0.1 and $0.0005 \mathrm{fs}$ as used in ref. 31 .

\section{Conclusion}

We presented an ab initio molecular dynamics simulation protocol based on MC-PDFT energies and gradients using the SHARC ${ }^{37-39}$ molecular dynamics package. We computed vertical excitation energies and performed population dynamics of photo-excited thioformaldehyde with MC-PDFT, and the results were compared to previous MS-CASPT2 results. ${ }^{24}$ While previous MS-CASPT2 SHARC dynamics were obtained only with the $(10,6)$ active space, but not with the larger $(12,10)$ active space, due to their computational expense, we could perform the MC-PDFT dynamics with both active spaces and obtained results in general agreement with fluorescence experiments. ${ }^{45-47}$ 
MC-PDFT allows us to perform excited-states dynamics with larger active spaces than with MS-CASPT2 and it gives encouraging results. We envision that MC-PDFT will become a method of choice for excited-states dynamics when the multi-state gradients become available.

\section{Supporting Information}

See the Supporting Information for coordinates of optimized structures, electronic energies of optimized thioformaldehyde structures, molecular orbitals for the $(10,6)$ active space, Wigner distribution frequencies, SHARC input files, discussion of timings, and a potential energy scan along the $\mathrm{C}-\mathrm{S}$ bond stretching coordinate.

\section{Acknowledgments}

The authors are grateful to Matthew Hermes, Yinan Shu, Dihua Wu, and Chen Zhou for many

helpful discussions of related topics. This work was supported by the National Science

Foundation under grant CHE-2054723. The computational resources for this project were provided by the University of Chicago Research Computing Center (RCC).

\section{References}

1. Marx, D.; Hutter, J. Ab Initio Molecular Dynamics: Basic Theory and Advanced Methods; Cambridge University Press, 2009.

2. Wang, I. S. Y.; Karplus, M. Dyanmics of organic reactions. Journal of the American Chemical Society. 1973, 95, 8160-8164.

3. Malcolme-Lawes, D. J. Dynamics of some hydrogen isotopic exchange reactions at high energies. Journal of the Chemical Society, Faraday Transactions 2: Molecular and Chemical Physics. 1975, 71, 1183-1199.

4. Leforestier, C. Classical trajectories using the full ab initio potential energy surface $\mathrm{H}^{-}+\mathrm{CH}_{4} \rightarrow \mathrm{CH}_{4}+\mathrm{H}^{-}$. J. Chem. Phys. 1978, 68, 4406-4410.

5. Baldridge, K. K.; Gordon, M. S.; Steckler, R.; Truhlar, D. G. Ab initio reaction paths and direct dynamics calculations. J. Phys. Chem. 1989, 93, 5107-5119.

6. Wentzcovitch, R. M.; Martins, J. First principles molecular dynamics of Li: Test of a new algorithm. Solid State Communications. 1991, 78, 831-834.

7. Barnett, R. N.; Landman, U. Born-Oppenheimer molecular-dynamics simulations of finite systems: Structure and dynamics of $\left(\mathrm{H}_{2} \mathrm{O}\right)_{2}$. Physical Review B. 1993, 48, 2081-2097.

8. Gibson, D. A.; Ionova, I. V.; Carter, E. A. A comparison of Car-Parrinello and BornOppenheimer generalized valence bond molecular dynamics. Chem. Phys. Lett. 1995, 240, 261267.

9. Cheng, H. P. The motion of protons in water-ammonia clusters. J. Chem. Phys. 1996, 105, 6844-6855.

10. Termath, V.; Sauer, J. Ab initio molecular dynamics simulation of $\mathrm{H}_{5} \mathrm{O}_{2}{ }^{+}$and $\mathrm{H}_{7} \mathrm{O}_{3}{ }^{+}$gas phase clusters based on density functional theory. Mol. Phys. 1997, 91, 963-975. 
11. Born, M.; Oppenheimer, R. Zur Quantentheorie der Molekeln. Ann. Phys. 1927, 389, 457484.

12. Bjerre, A.; Nikitin, E. E. Energy transfer in collisions of an excited sodium atom with a nitrogen molecule. Chem. Phys. Lett. 1967, 1, 179-181.

13. Tully, J. C.; Preston, R. K. Trajectory Surface Hopping Approach to Nonadiabatic Molecular Collisions: The Reaction of $\mathrm{H}^{+}$with $\mathrm{D}_{2}$. J. Chem. Phys. 1971, 55, 562-572.

14. Chapman, S.; Preston, R. K. Nonadiabatic molecular collisions: Charge exchange and chemical reaction in the $\mathrm{Ar}^{+}+\mathrm{H}_{2}$ system. J. Chem. Phys. 1974, 60, 650-659.

15. Kuntz, P. J.; Kendrick, J.; Whitton, W. N. Surface-hopping trajectory calculations of collision-induced dissociation processes with and without charge transfer. Chem. Phys. 1979, 38, 147-160.

16. Truhlar, D. G.; Duff, J. W.; Blais, N. C.; Tully, J. C.; Garrett, B. C. The quenching of $\mathrm{Na}\left(3{ }^{2} \mathrm{P}\right)$ by $\mathrm{H}_{2}$ : Interactions and dynamics. J. Chem. Phys. 1982, 77, 764-776.

17. González, L.; Lindh, R. Quantum Chemistry and Dynamics of Excited States: Methods and Applications; John Wiley \& Sons, 2021.

18. Truhlar, D. G.; Mead, C. A. Relative likelihood of encountering conical intersections and avoided intersections on the potential energy surfaces of polyatomic molecules. Physical Review A. 2003, 68, 032501.

19. González, L.; Escudero, D.; Serrano-Andrés, L. Progress and Challenges in the Calculation of Electronic Excited States. ChemPhysChem. 2012, 13, 28-51.

20. Roos, B. O.; Taylor, P. R.; Sigbahn, P. E. M. A complete active space SCF method (CASSCF) using a density matrix formulated super-CI approach. Chem. Phys. 1980, 48, 157-173.

21. Andersson, K.; Malmqvist, P. A.; Roos, B. O.; Sadlej, A. J.; Wolinski, K. Second-order perturbation theory with a CASSCF reference function. J. Phys. Chem. 1990, 94, 5483-5488.

22. Finley, J.; Malmqvist, P.-Å.; Roos, B. O.; Serrano-Andrés, L. The multi-state CASPT2 method. Chem. Phys. Lett. 1998, 288, 299-306.

23. Nishimoto, Y. Analytic gradients for restricted active space second-order perturbation theory (RASPT2). J. Chem. Phys. 2021, 154, 194103.

24. Mai, S.; Atkins, A. J.; Plasser, F.; González, L. The Influence of the Electronic Structure Method on Intersystem Crossing Dynamics. The Case of Thioformaldehyde. J. Chem. Theory Comput. 2019, 15, 3470-3480.

25. Li Manni, G.; Carlson, R. K.; Luo, S.; Ma, D.; Olsen, J.; Truhlar, D. G.; Gagliardi, L. Multiconfiguration Pair-Density Functional Theory. J. Chem. Theory Comput. 2014, 10, 36693680.

26. Gagliardi, L.; Truhlar, D. G.; Li Manni, G.; Carlson, R. K.; Hoyer, C. E.; Bao, J. L. Multiconfiguration Pair-Density Functional Theory: A New Way To Treat Strongly Correlated Systems. Acc. Chem. Res. 2017, 50, 66-73.

27. Ghosh, S.; Verma, P.; Cramer, C. J.; Gagliardi, L.; Truhlar, D. G. Combining Wave Function Methods with Density Functional Theory for Excited States. Chem. Rev. 2018, 118, 7249-7292.

28. Sand, A. M.; Hoyer, C. E.; Sharkas, K.; Kidder, K. M.; Lindh, R.; Truhlar, D. G.; Gagliardi, L. Analytic Gradients for Complete Active Space Pair-Density Functional Theory. J. Chem. Theory Comput. 2018, 14, 126-138.

29. Scott, T. R.; Hermes, M. R.; Sand, A. M.; Oakley, M. S.; Truhlar, D. G.; Gagliardi, L. Analytic gradients for state-averaged multiconfiguration pair-density functional theory. J. Chem. Phys. 2020, 153, 014106. 
30. Scott, T. R.; Oakley, M. S.; Hermes, M. R.; Sand, A. M.; Lindh, R.; Truhlar, D. G.; Gagliardi, L. Analytic gradients for multiconfiguration pair-density functional theory with density fitting: Development and application to geometry optimization in the ground and excited states. $J$. Chem. Phys. 2021, 154, 074108.

31. Marín, M. d. C.; De Vico, L.; Dong, S. S.; Gagliardi, L.; Truhlar, D. G.; Olivucci, M. Assessment of MC-PDFT Excitation Energies for a Set of QM/MM Models of Rhodopsins. $J$. Chem. Theory Comput. 2019, 15, 1915-1923.

32. Kolesov, G.; Grånäs, O.; Hoyt, R.; Vinichenko, D.; Kaxiras, E. Real-Time TD-DFT with Classical Ion Dynamics: Methodology and Applications. J. Chem. Theory Comput. 2016, 12, 466476.

33. Parker, S. M.; Roy, S.; Furche, F. Multistate hybrid time-dependent density functional theory with surface hopping accurately captures ultrafast thymine photodeactivation. Phys. Chem. Chem. Phys. 2019, 21, 18999-19010.

34. Martínez, T. J. Insights for Light-Driven Molecular Devices from Ab Initio Multiple Spawning Excited-State Dynamics of Organic and Biological Chromophores. Acc. Chem. Res. 2006, 39, 119-126.

35. Levine, B. G.; Coe, J. D.; Virshup, A. M.; Martínez, T. J. Implementation of ab initio multiple spawning in the Molpro quantum chemistry package. Chem. Phys. 2008, 347, 3-16.

36. Mai, S.; Marquetand, P.; Richter, M.; González-Vázquez, J.; González, L. Singlet and Triplet Excited-State Dynamics Study of the Keto and Enol Tautomers of Cytosine. ChemPhysChem. 2013, 14, 2920-2931.

37. Richter, M.; Marquetand, P.; González-Vázquez, J.; Sola, I.; González, L. SHARC: ab Initio Molecular Dynamics with Surface Hopping in the Adiabatic Representation Including Arbitrary Couplings. J. Chem. Theory Comput. 2011, 7, 1253-1258.

38. Mai, S.; Marquetand, P.; González, L. A general method to describe intersystem crossing dynamics in trajectory surface hopping. Int. J. Quantum Chem. 2015, 115, 1215-1231.

39. Mai, S.; Marquetand, P.; González, L. Nonadiabatic dynamics: The SHARC approach. WIREs Comput Mol Sci. 2018, 8, e1370.

40. Fdez. Galván, I.; Vacher, M.; Alavi, A.; Angeli, C.; Aquilante, F.; Autschbach, J.; Bao, J. J.; Bokarev, S. I.; Bogdanov, N. A.; Carlson, R. K.; Chibotaru, L. F.; Creutzberg, J.; Dattani, N.; Delcey, M. G.; Dong, S. S.; Dreuw, A.; Freitag, L.; Frutos, L. M.; Gagliardi, L.; Gendron, F.; Giussani, A.; González, L.; Grell, G.; Guo, M.; Hoyer, C. E.; Johansson, M.; Keller, S.; Knecht, S.; Kovačević, G.; Källman, E.; Li Manni, G.; Lundberg, M.; Ma, Y.; Mai, S.; Malhado, J. P.; Malmqvist, P. Å; Marquetand, P.; Mewes, S. A.; Norell, J.; Olivucci, M.; Oppel, M.; Phung, Q. M.; Pierloot, K.; Plasser, F.; Reiher, M.; Sand, A. M.; Schapiro, I.; Sharma, P.; Stein, C. J.; Sørensen, L. K.; Truhlar, D. G.; Ugandi, M.; Ungur, L.; Valentini, A.; Vancoillie, S.; Veryazov, V.; Weser, O.; Wesołowski, T. A.; Widmark, P.-O.; Wouters, S.; Zech, A.; Zobel, J. P.; Lindh, R. OpenMolcas: From Source Code to Insight. J. Chem. Theory Comput. 2019, 15, 5925-5964.

41. Minnesota-Chicago OpenMolcas Homepage,. https://comp.chem.umn.edu/openmolcas (accessed Aug. 25, 2021).

42. Steer, R. P. Structure and decay dynamics of electronic excited states of thiocarbonyl compounds. Reviews of Chemical Intermediates. 1981, 4, 1-41.

43. El-Sayed, M. A. Triplet state. Its radiative and nonradiative properties. Acc. Chem. Res. 1968, $1,8-16$. 
44. Zhang, L.; Shu, Y.; Sun, S.; Truhlar, D. G. Direct coherent switching with decay of mixing for intersystem crossing dynamics of thioformaldehyde: The effect of decoherence. J. Chem. Phys. 2021, 154, 094310.

45. Clouthier, D. J.; Ramsay, D. A. The Spectroscopy of Formaldehyde and Thioformaldehyde. Annu. Rev. Phys. Chem. 1983, 34, 31-58.

46. Kawasaki, M.; Kasatani, K.; Ogawa, Y.; Sato, H. Spectra and emission lifetimes of $\mathrm{H}_{2} \mathrm{CS}\left(\tilde{\mathrm{A}}^{1} \mathrm{~A}^{2}\right)$. Chem. Phys. 1983, 74, 83-88.

47. Moule, D. C.; Lim, E. C. Highly Varying Photophysical Properties of Thiocarbonyls: Validation of Fundamental Theoretical Concepts of Electronic Radiationless Transitions. J. Phys. Chem. A. 2002, 106, 3072-3076.

48. $\quad$ Lischka, H.; Shepard, R.; Pitzer, R. M.; Shavitt, I.; Dallos, M.; Müller, T.; Szalay, P. G.; Seth, M.; Kedziora, G. S.; Yabushita, S.; Zhang, Z. High-level multireference methods in the quantum-chemistry program system COLUMBUS: Analytic MR-CISD and MR-AQCC gradients and MR-AQCC-LRT for excited states, GUGA spin-orbit CI and parallel CI density. Phys. Chem. Chem. Phys. 2001, 3, 664-673.

49. Pople, J. A.; Seeger, R.; Krishnan, R. Variational configuration interaction methods and comparison with perturbation theory. Int. J. Quantum Chem. 1977, 12, 149-163.

50. Dunning, T. H. Gaussian basis sets for use in correlated molecular calculations. I. The atoms boron through neon and hydrogen. J. Chem. Phys. 1989, 90, 1007-1023.

51. Reiher, M.; Wolf, A. Exact decoupling of the Dirac Hamiltonian. I. General theory. $J$. Chem. Phys. 2004, 121, 2037-2047.

52. Reiher, M.; Wolf, A. Exact decoupling of the Dirac Hamiltonian. II. The generalized Douglas-Kroll-Hess transformation up to arbitrary order. J. Chem. Phys. 2004, 121, 10945-10956.

53. Nakajima, T.; Hirao, K. The Douglas-Kroll-Hess Approach. Chem. Rev. 2012, 112, 385402.

54. Malmqvist, P.-Å; Roos, B. O. The CASSCF state interaction method. Chem. Phys. Lett. 1989, 155, 189-194.

55. Malmqvist, P. Å; Roos, B. O.; Schimmelpfennig, B. The restricted active space (RAS) state interaction approach with spin-orbit coupling. Chem. Phys. Lett. 2002, 357, 230-240.

56. Fdez. Galván, I.; Delcey, M. G.; Pedersen, T. B.; Aquilante, F.; Lindh, R. Analytical StateAverage Complete-Active-Space Self-Consistent Field Nonadiabatic Coupling Vectors: Implementation with Density-Fitted Two-Electron Integrals and Application to Conical Intersections. J. Chem. Theory Comput. 2016, 12, 3636-3653.

57. Tully, J. C. Molecular dynamics with electronic transitions. J. Chem. Phys. 1990, 93, 10611071.

58. Meyer, H. D.; Miller, W. H. A classical analog for electronic degrees of freedom in nonadiabatic collision processes. J. Chem. Phys. 1979, 70, 3214-3223.

59. Topaler, M. S.; Allison, T. C.; Schwenke, D. W.; Truhlar, D. G. What is the best semiclassical method for photochemical dynamics of systems with conical intersections? J. Chem. Phys. 1998, 109, 3321-3345.

60. Granucci, G.; Persico, M.; Zoccante, A. Including quantum decoherence in surface hopping. J. Chem. Phys. 2010, 133, 134111.

61. Plasser, F.; Mai, S.; Fumanal, M.; Gindensperger, E.; Daniel, C.; González, L. Strong Influence of Decoherence Corrections and Momentum Rescaling in Surface Hopping Dynamics of Transition Metal Complexes. J. Chem. Theory Comput. 2019, 15, 5031-5045. 
62. Zhu, C.; Nangia, S.; Jasper, A. W.; Truhlar, D. G. Coherent switching with decay of mixing: An improved treatment of electronic coherence for non-Born-Oppenheimer trajectories. J. Chem. Phys. 2004, 121, 7658-7670.

63. Lengsfield, B. H.; Saxe, P.; Yarkony, D. R. On the evaluation of nonadiabatic coupling matrix elements using SA-MCSCF/CI wave functions and analytic gradient methods. I. J. Chem. Phys. 1984, 81, 4549-4553.

64. Lengsfield Iii, B. H.; Yarkony, D. R. Nonadiabatic Interactions Between Potential Energy Surfaces: Theory and Applications. Advances in Chemical Physics. 1992, 1-71.

65. Wigner, E. On the Quantum Correction For Thermodynamic Equilibrium. Phys. Rev. 1932, 40, 749-759.

66. Barbatti, M.; Granucci, G.; Persico, M.; Ruckenbauer, M.; Vazdar, M.; Eckert-Maksić, M.; Lischka, H. The on-the-fly surface-hopping program system Newton-X: Application to ab initio simulation of the nonadiabatic photodynamics of benchmark systems. J. Photochem. Photobiol., A. 2007, 190, 228-240.

67. Granucci, G.; Persico, M.; Toniolo, A. Direct semiclassical simulation of photochemical processes with semiempirical wave functions. J. Chem. Phys. 2001, 114, 10608-10615.

68. Celani, P.; Werner, H.-J. Multireference perturbation theory for large restricted and selected active space reference wave functions. J. Chem. Phys. 2000, 112, 5546-5557.

69. Curchod, B. F. E.; Rauer, C.; Marquetand, P.; González, L.; Martínez, T. J. Communication: GAIMS - Generalized Ab Initio Multiple Spawning for both internal conversion and intersystem crossing processes. J. Chem. Phys. 2016, 144, 101102.

70. Sand, A. M.; Hoyer, C. E.; Truhlar, D. G.; Gagliardi, L. State-interaction pair-density functional theory. J. Chem. Phys. 2018, 149, 024106.

71. Bao, J. J.; Zhou, C.; Varga, Z.; Kanchanakungwankul, S.; Gagliardi, L.; Truhlar, D. G. Multi-state pair-density functional theory. Faraday Discuss. 2020, 224, 348-372.

72. Bao, J. J.; Zhou, C.; Truhlar, D. G. Compressed-State Multistate Pair-Density Functional Theory. J. Chem. Theory Comput. 2020, 16, 7444-7452. 


\section{For Table of Contents Only}

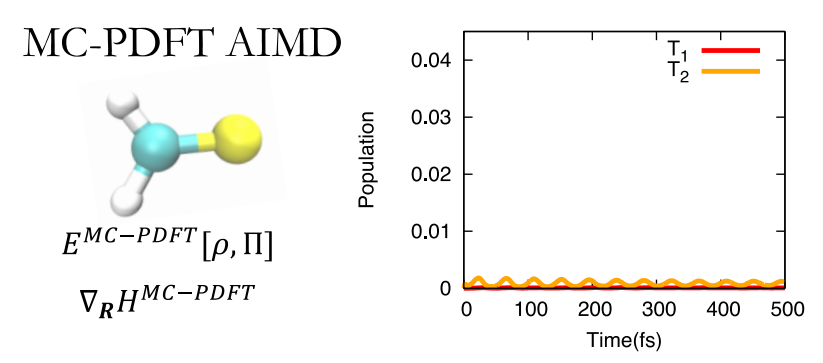

